\title{
Rapid Characterization of Oxygen-Evolving Electrocatalyst Spot Arrays by the Substrate Generation/Tip Collection Mode of Scanning Electrochemical Microscopy with Decreased $\mathrm{O}_{2}$ Diffusion Layer Overlap
}

\author{
Alessandro Minguzzi, ${ }^{\dagger}, \#$ Dario Battistel, ${ }^{\ddagger}$ Joaquin Rodríguez-López, ${ }^{\S}$ Alberto Vertova, ${ }^{\dagger, \#, \otimes}$ \\ Sandra Rondinini, ${ }^{\dagger, \#, \otimes}$ Allen J. Bard," and Salvatore Daniele ${ }^{\perp}$ \\ ${ }^{\dagger}$ Dipartimento di Chimica, Università degli Studi di Milano, via Golgi 19, 20133 Milano, Italy \\ ”Dipartimento di Scienze Ambientali Informatica a Statistica, Università “Ca’ Foscari” Venezia, Calle Larga, S. Marta 2137, I-30123 \\ Venice, Italy \\ ${ }^{\S}$ Department of Chemistry, University of Illinois at Urbana-Champaign, 600 South Mathews Avenue, Urbana, Illinois 61801, United \\ States \\ "Center for Electrochemistry, Department of Chemistry and Biochemistry, The University of Texas at Austin, Austin, Texas 78712, \\ United States \\ ${ }^{\perp}$ Dipartimento di Scienze Molecolari e Nanosistemi, Università “Ca’ Foscari” Venezia, Calle Larga, S. Marta 2137, I-30123 Venice, \\ Italy \\ \#Istituto Nazionale di Scienza e Tecnologia dei Materiali, via Giusti 9, 50121 Firenze, Italy \\ ${ }^{\otimes}$ CNR-ISTM, Istituto di Scienze e Tecnologie Molecolari, I-20133 Milan, Italy
}

ABSTRACT: A simple approach for the screening of oxygen evolution reaction (OER) electrocatalyst arrays by scanning electrochemical microscopy (SECM) in the substrate generation/tip collection (SG/TC) mode is described. The methodology is based on the application of a series (9-10 replicates) of double-potential steps to a catalytically active substrate electrode, which is switched between potentials where it displays OER activity and inactivity. With an SECM tip coaligned to a given electrocatalyst spot, the dual potential step is applied for a relatively short time in order to restrict the growth of the resulting $\mathrm{O}_{2}$ diffusion layer. The SECM is then able to measure the $\mathrm{O}_{2}$ produced while the potential sequence prevents the overlap of the diffusion layer from neighboring spots. With this approach, each spot of material in an array of Ir:Sn oxide compositions (disk shaped, about $150 \mu \mathrm{m}$ radius) was examined independently at a constant distance. The method was tested for a series of oxygen evolution catalysts made of $\mathrm{SnO}_{2}-\mathrm{IrO}_{2}$ mixtures, with compositions varying between Ir:Sn 100:0 to Ir:Sn 0:100. Optimal conditions for avoiding overlapping of the diffusion profiles generated at each spot of the substrate were evaluated by digital simulation. The results obtained for the activity of $\mathrm{SnO}_{2}-\mathrm{IrO}_{2}$ mixtures using this new technique were validated by comparison to reported results using SECM and other techniques.

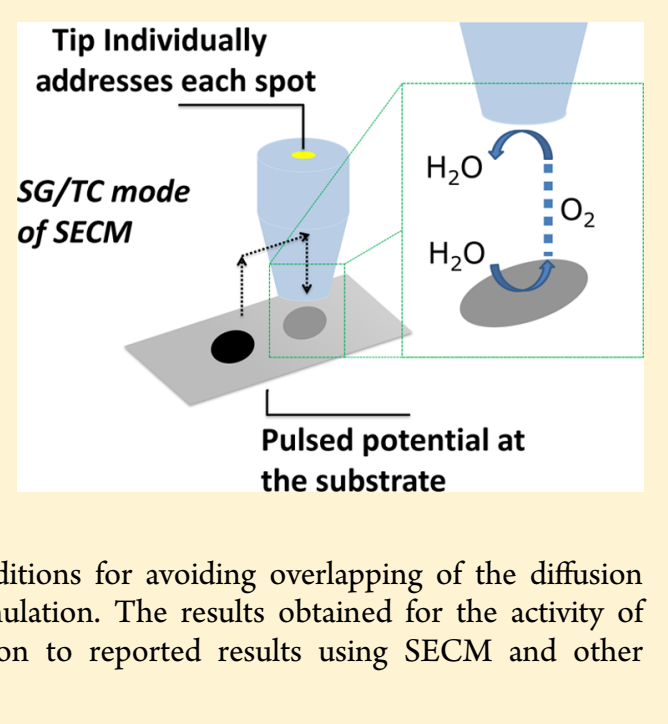

Tip Individually

addresses each spot

SG/TC mode

Pulsed potential at

the substrate

\section{INTRODUCTION}

Electrocatalyst screening techniques based on scanning electrochemical microscopy (SECM) display unparalleled versatility in the combinatorial evaluation of electrocatalysts and photoelectrocatalysts. ${ }^{1-12}$ These techniques allow one to quickly obtain mechanistic information about a set of materials in a well-controlled compositional design space and to compare the performance of different samples under exactly the same reaction conditions. Typically, the screening involves using an SECM tip that addresses a small part of the electrode in close proximity and records an electrochemical signal resulting from sample activity. This signal is transduced spatially into an
SECM image. The activities of the spots are then evaluated on the basis of a color code/scale that is proportional to the signal intensity. There are different SECM modes to measure the electrocatalytic activity on a substrate and include: feedback, ${ }^{3}$ tip generation/substrate collection (TG/SC), ${ }^{1,2,13}$ shielding (redox competition), ${ }^{14,15}$ and substrate generation/tip collection (SG/TC) mode. ${ }^{16-19}$ Here, we will focus on the SG/TC mode, which has been applied extensively to the quantification

Received: October 23, 2014

Revised: December 24, 2014

Published: January 19, 2015 


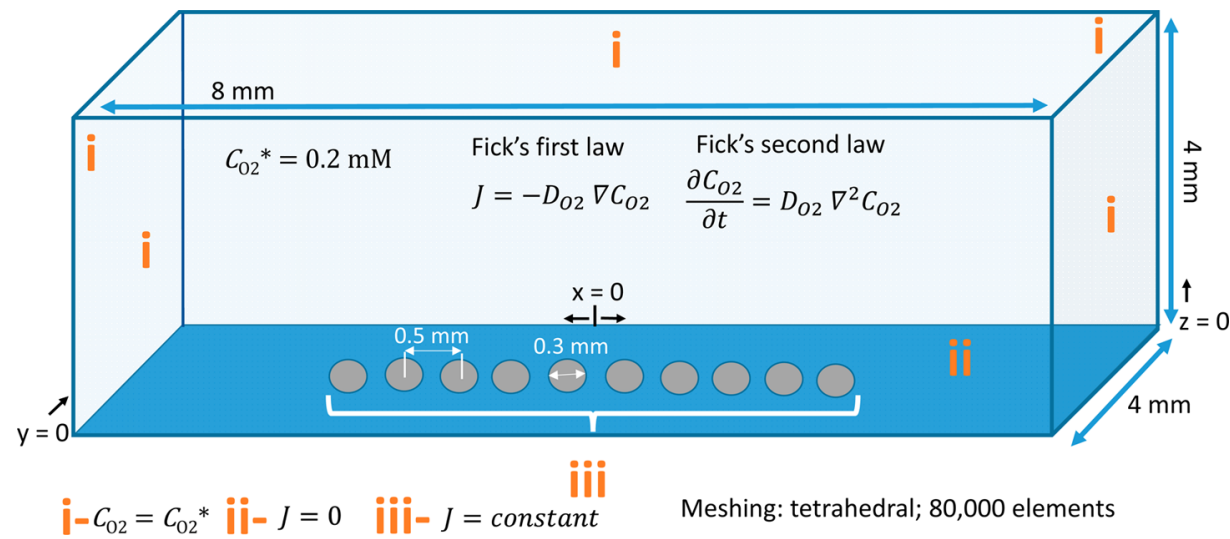

Figure 1. Schematic design of geometry, subdomains, and boundary conditions (marked in orange) relevant to the 3D ( $x, y, z$ space) digital simulations used in this work. $J$ indicates the diffusive flux of $\mathrm{O}_{2}$ and $D$ its diffusion coefficient.

of $\mathrm{H}_{2} \mathrm{O}_{2}$ from catalytic and electrocatalytic systems ${ }^{16,19-24}$ and for gas-evolving reactions ${ }^{25}$ such as the chlorine evolution reaction ${ }^{6,26}$ and the oxygen evolution reaction. ${ }^{27,28}$

In the SG/TC mode applied to the oxygen evolution reaction, the spots of the array are biased at a potential where water is oxidized at a steady state. The SECM tip acts as an amperometric sensor that collects the $\mathrm{O}_{2}$ generated from each spot as the tip is moved in the XY plane. A problem that arises in this approach is the overlap of diffusion profiles of neighboring spots in the array. ${ }^{29}$ This point has been addressed in previous work, but specifically for the OER, a tip-shielding approach was used in order to investigate the optimum composition of $\mathrm{SnO}_{2}-\mathrm{IrO}_{2}$ catalysts. ${ }^{27}$ In this case, the tip was surrounded by a gold layer, deposited on its external wall, and used as a tip shield by applying a constant potential to reduce interfering oxygen from neighboring spots under mass transfer controlled conditions. While this approach is effective at reducing $\mathrm{O}_{2}$ diffusion layer overlap, it requires the fabrication of a special SECM tip.

In this work, we introduce a SECM transient method that does not require a special probe. Here, the diffusion layer growth, which is generated at each catalyst spot, is limited by reducing the reaction time and by the application of a series potential pulses at the substrate. A regular gold tip acts as an amperometric sensor for collecting the generated $\mathrm{O}_{2}$. The validity of this approach was tested by screening the activity of an array of $\mathrm{SnO}_{2}-\mathrm{IrO}_{2}$ composites toward the oxygen evolution reaction but is in principle extendable to any multiple combination of materials to be studied as electrocatalysts/ photoelectrocatalysts for gas evolution. Notwithstanding the application of a pulsed substrate potential profile for the correct detection of the substrate product has already been reported in the literature, ${ }^{19,22}$ the method here proposed represents the first application to electrocatalysts screening and particularly in the case of oxygen evolution reaction (OER). We obtain excellent agreement between this new methodology and previously reported SECM screening results, thus allowing a direct comparison and confirming the goodness of our approach.

\section{EXPERIMENTAL METHODS}

2.1. Sample Preparation. In the considered array (SnIr1), spots consisting of $\mathrm{Sn}_{1-x} \mathrm{Ir}_{x} \mathrm{O}_{2}$ mixed oxides in the nominal composition range from 0 to $100 \% \mathrm{IrO}_{2}$ were prepared in $10 \%$ $(\Delta x=0.1)$ increments. A solution of $0.1 \mathrm{M} \mathrm{IrCl}_{3} \cdot 3 \mathrm{H}_{2} \mathrm{O}$ in water: glycerol (3:1) was first dispensed on Ti foil $(1.5 \times 1.5$ $\mathrm{cm}, 0.75 \mathrm{~mm}$ thickness, $99.7 \%$ purity, Aldrich, St. Louis, MO, previously washed in water and sonicated in acetone) using a CHI 1550 picoliter dispenser ( $\mathrm{CH}$ Instruments, Austin, TX), with spot deposition parameters: pulse amplitude, $50 \mathrm{~V}$; pulse width, $15 \mu \mathrm{s}$; pulse period, $500 \mathrm{~ms}$; center to center distance, $500 \mu \mathrm{m}$, while the number of drops in each column were changed from 10 to 0 in the $y$ direction. Subsequently, a $0.1 \mathrm{M}$ solution of $\mathrm{SnCl}_{4} \cdot 5 \mathrm{H}_{2} \mathrm{O}$ (98\% purity Alfa Aesar, Ward Hill, $\mathrm{MA})$ in water:glycerol (3:1) was dispensed in the same way as for the Ir precursor. To keep the total moles of the two components constant on every spot, the number of drops was varied between 0 and 10 , such that $11 \mathrm{Sn}_{1-x} \mathrm{Ir}_{x} \mathrm{O}_{2}$ spots were deposited with nominal compositions ranging from $x=1$ $\left(100 \% \mathrm{IrO}_{2}\right)$ to $x=0\left(100 \% \mathrm{SnO}_{2}\right)$ with steps of $\Delta x=0.1$. The array consisted of a $11 \times 3$ grid (three equivalent spot lines to study reproducibility). The sample was vortexed for $10 \mathrm{~min}$ and immediately exposed to gaseous $\mathrm{NH}_{3}(1 \mathrm{~atm})$ for $1 \mathrm{~h}$. The sample was aged in air for $24 \mathrm{~h}$ and dried for $1 \mathrm{~h}$ at $180{ }^{\circ} \mathrm{C}$ (after a $5{ }^{\circ} \mathrm{C} / \mathrm{min}$ ramp) in a tube furnace under an Ar flow. Finally, the arrays were annealed at $500{ }^{\circ} \mathrm{C}$ for $2 \mathrm{~h}$ in oxygen ( 1 atm). During the cooling period, samples were kept under Ar. This procedure yielded spots with a diameter of $300 \mu \mathrm{m}$ on average.

2.2. Tip Preparation. A $100 \mu \mathrm{m}$ diameter gold wire (Goodfellow, 99.99\% purity) was sealed into a flint glass capillary o.d./i.d. 1.5/0.75 mm (no. 27-37-1, Frederik Haer \& Co., Bowdoinham, ME) under vacuum. One end was then polished with 600 mesh sandpaper (Buehler, Lake Bluff, IL) until the metal disk was exposed and then smoothed with 1200mesh abrasive $\mathrm{SiC}$ paper and alumina suspensions in water down to $0.3 \mu \mathrm{m}$ (Buehler). Finally, the tip was sharpened, reaching an RG (ratio of glass to metal radius) of 4 .

Silver-epoxy (Epotek $\mathrm{H}_{2} \mathrm{OE}$, Epoxy Technology, Billerica, MA) cured overnight at $100{ }^{\circ} \mathrm{C}$ was used to make electric contact between the gold wire and a copper wire (used as a current collector). Finally, the tip surface was polished to remove contaminants deposited on the gold surface during this procedure with a $0.3 \mu \mathrm{m}$ alumina powder suspension on an adhesive cloth supported on a hard, smooth surface.

2.3. Scanning Electrochemical Microscopy. All measurements were performed in $0.5 \mathrm{M} \mathrm{H}_{2} \mathrm{SO}_{4}$ aqueous solution in equilibrium with air. A tungsten wire was used as counter electrode, and a reversible hydrogen electrode as the reference electrode. The RHE was made with a Pt wire in a separate 


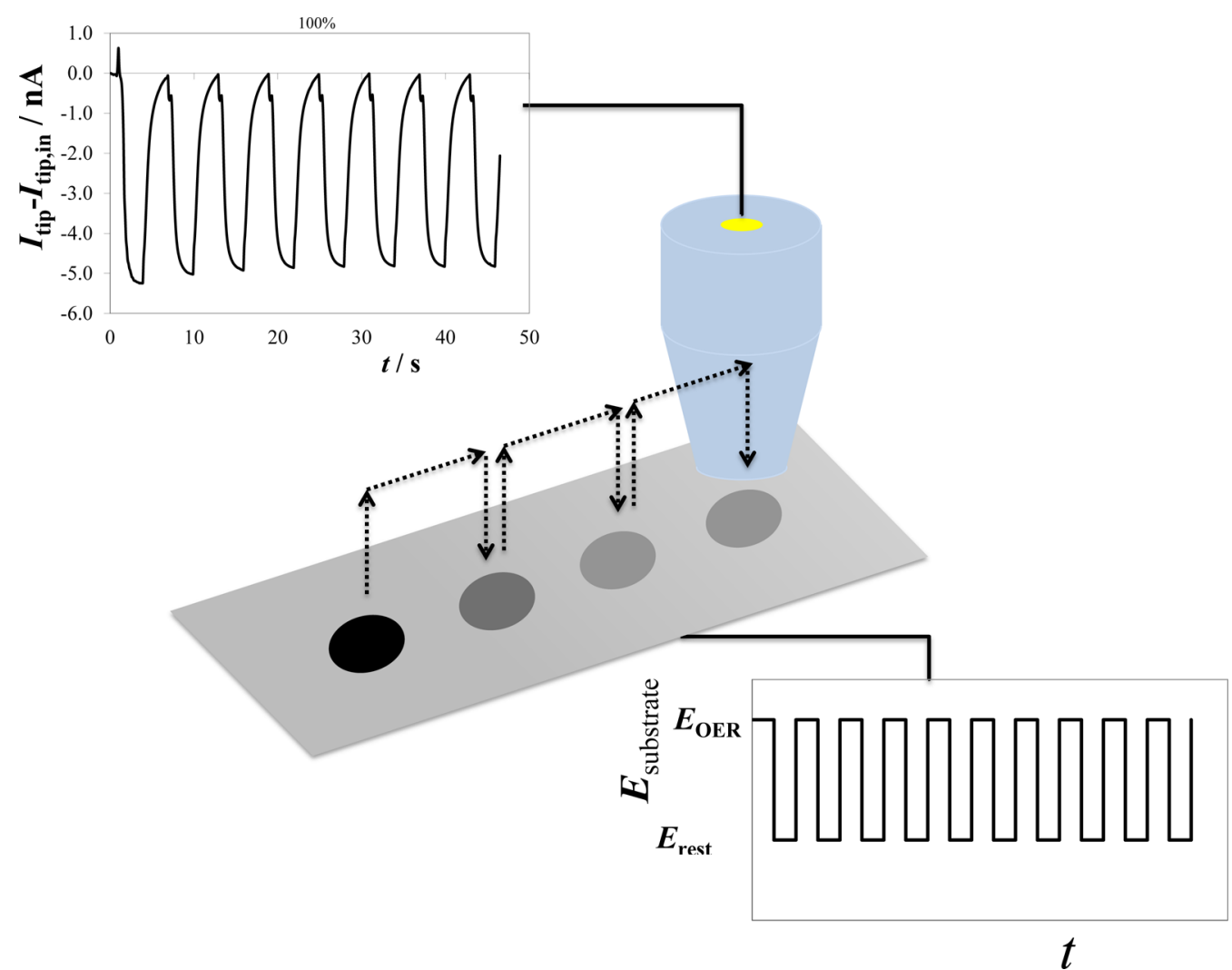

Figure 2. Pictorial representation of the interrogation of single spots (from black to gray, indicating different chemical compositions) by the tip, whose current $\left(I_{\text {tip }}\right)$ oscillates according to the double pulse potential profile applied at the substrate $\left(E_{\text {substrate }}\right)$.

borosilicate capillary glass with a soft glass cracked junction filled with $0.5 \mathrm{M} \mathrm{H}_{2} \mathrm{SO}_{4}$ and saturated with 1 atm $\mathrm{H}_{2}$ obtained by electrogeneration. The sample substrate was placed on the top of a rigid $\mathrm{Cu}$ plate laid on a flat acrylic base to provide mechanical support. A PTFE cell with a $9 \mathrm{~mm}$ diameter orifice in the center was tightened to the acrylic base using an o-ring. The SECM micropositioning device consisted of a set of three stepper motor stages with a $0.1 \mathrm{~mm}$ resolution (MICOS) with optical encoder (ZEISS), and the motion was controlled by a closed loop motion controller board PCI-7324 (National Instruments). The data acquisition was performed by a PCI6035E Multifunction I/O with a Lab View software (National Instruments). The substrate and tip potentials were driven by using a PAR 175 function generator (associated with a current amplifier Keithley 428) and a CH Instrument $1222 \mathrm{~A}$, respectively.

Because in the double-pulse potential experiments described here every spot is addressed individually through an approach curve, the substrate tilt was not thoroughly corrected. Instead, we used negative feedback approach curves using $\mathrm{O}_{2}$ reduction at the tip to roughly correct the tilt by performing approach curves in different points of the sample. Preliminary line scans performed over the sample allowed us to assign the position of each spot in the array. Before each series of measurements, the tip was brought over the desired spot at a distance of $20 \mu \mathrm{m}$ and kept at the working potential of $-0.1 \mathrm{~V}$ vs RHE, where oxygen reduction to water takes place. This was done by means of $1 \mu \mathrm{m} \mathrm{s}^{-1}$ approach curves. The distance was set by stopping the approach curve at the predicted tip current calculated using empirical negative feedback equations. ${ }^{30}$ A stable current was achieved after $1 \mathrm{~min}$. Afterward, the substrate potential was varied following a square-wave profile, as displayed in inset in
Figure 1, between oxygen evolution potential $\left(E_{\mathrm{OER}}\right)$ and a rest potential $\left(E_{\text {rest }}\right)$ where no significant electrode reaction occurred.

2.4. Simulations. Digital simulations were performed using the Comsol Multiphysics software 3.5a, which uses the finite element method to solve for the required diffusion problem. A scheme of the simulation geometry, boundary conditions, and the governing equation is reported in Figure 1. In short, we modeled the OER-inactive substrate as with a zero-flux boundary condition. Over this surface, microelectrodes of 150 $\mu \mathrm{m}$ in radius and spaced with a center-to-center distance of 500 $\mu \mathrm{m}$, which represented the electrocatalytic spots, were modeled as surfaces with a constant flux output. This flux is specified in the discussion section and represents the current generated during the activity pulse at $E_{\mathrm{OER}}$. It was changed to zero when the spot was held at rest, representing the zero output at $E_{\text {rest }}$.

The flux of molecules diffusing away from the substrate caused the time-dependent growth of a diffusion layer which displayed different concentration profiles depending on the specified activity (flux). The SECM response for an arbitrary SECM tip was linearly proportional to the specified flux, so a direct comparison between tip current and relative spot activity can be drawn. The period of the square wave could be varied to allow different degrees of overlap between the diffusion layers created by the spots. The optimum period for the double pulse sequence was obtained by measuring the tip current over one spot in the presence of activity of neighboring spots but inactivity from the addressed spot. A threshold was defined such that the time-dependent flux coming from the neighboring spots, which grows with time, did not account for more than $3 \%$ of the total flux from the neighboring spot over a total of 10 cycles. This criterion both takes into account that a low overall 
interfering signal is obtained, and that time-dependent changes due to the imposed waveform and any initial conditions are absorbed by this error tolerance.

\section{RESULTS AND DISCUSSION}

3.1. Optimization of the Potential Wave-Form at the Substrate and Current Response at the Tip. The approach employed here consists in pulsing the substrate potential between $E_{\mathrm{OER}}$ more positive than the equilibrium potential of the oxygen evolution reaction (i.e., $1.23 \mathrm{~V}$ vs RHE), and $E_{\text {rest }}$ a potential at which the spots are electrochemically inactive. For $\mathrm{Sn}_{1-x} \mathrm{Ir}_{x} \mathrm{O}_{2}$, a suitable potential found for $E_{\mathrm{OER}}$ was $1.4 \mathrm{~V}$ vs RHE. This activation is enough to provide a measurable $\mathrm{O}_{2}$ production, while avoiding bubble formation, and $E_{\text {rest }}$ which was varied between 1.2 and $0.9 \mathrm{~V}$ vs RHE. In the meantime, the tip was biased at a constant potential of $-0.1 \mathrm{~V}$ vs RHE, during the entire series of pulses, so that oxygen was reduced at a rate limited by mass transfer. The tip signal thus oscillates according to the substrate potential, as is shown in inset of the scheme displayed in Figure 2.

The optimal pulse length was established by digital simulation, varying the step pulse from 1 to 100 s. Figure 3

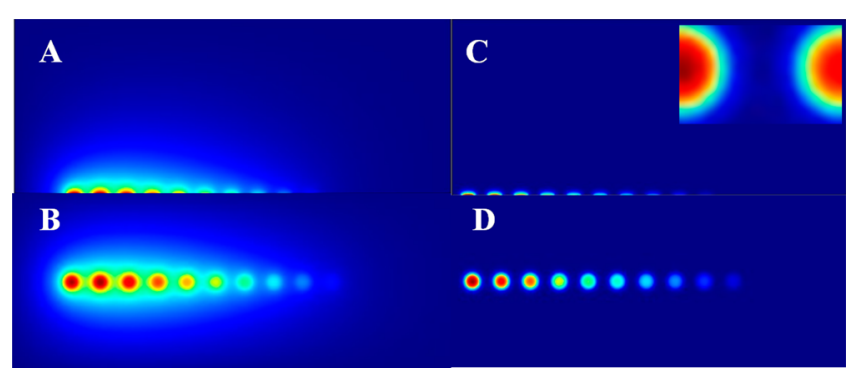

Figure 3. Digital simulation of a 10 spot array. A constant flux, corresponding to the following currents, comes out of each spot: from left to right: $0.2,0.18,0.16,0.14,0.12,0.1,0.08,0.06,0.04,0.02 \mu \mathrm{A}$ $\mathrm{cm}^{-2}$ is maintained for $100 \mathrm{~s}$ (A and B) or for only $3 \mathrm{~s}(\mathrm{C}$ and $\mathrm{D})$. Each picture represents the $2 \mathrm{D}$ oxygen concentration profiles in the $x-z$ plane (A and $\mathrm{C}$, the $y$ is set in correspondence to the spot line center) or in the in the $x-y$ one $(\mathrm{B}, \mathrm{D}, z=0)$. The inset is a magnification of the two most active spots of $\mathrm{D}$.

contrasts two sets of diffusion profiles obtained by applying 100 $\mathrm{s}$ (Figure 3A,B) and $3 \mathrm{~s}$ (Figure 3C,D) to a series of 10 spots that recreate an experimental array of $\mathrm{Sn}_{1-x} \mathrm{Ir}_{x} \mathrm{O}_{2}$ as described in the Experimental Section. These spots produce a progressively decreasing flux of oxygen when moving from an "Ir rich" spot to a "Sn rich" spot, thus roughly mimicking the actual activity of the array. The diffusion profiles in Figure 3A,B, where the spots are kept active for the longer time of $100 \mathrm{~s}$, overlap significantly and interfere with an accurate determination of the $\mathrm{O}_{2}$ concentration above the addressed spot. This causes the measurement to not be representative of the spot reaction rate. In contrast, allowing for only $3 \mathrm{~s}$ of operation as is done in Figure 3C,D yields a well-resolved diffusion profile that reflects each spot's activity. The latter value was therefore chosen as optimal pulse length for all measurements reported in this work. This result is consistent with a simple evaluation of the characteristic diffusion time between two electrocatalytic spots. Assuming a center-to-center distance of $500 \mu \mathrm{m}$ and a radius of $150 \mu \mathrm{m}$ per spot, the edge to edge distance per spot is $200 \mu \mathrm{m}$. The characteristic time can be calculated using $\tau=d^{2} /$ $2 D$ where $d$ is the edge-to-edge distance and $D$ is the diffusion coefficient, in this case oxygen in acidic media, $D=1.36 \times 10^{-5}$ $\mathrm{cm}^{2} / \mathrm{s}$. In this case, $\tau=14.7$, and its half, about $7 \mathrm{~s}$, represents the time needed for two diffusion profiles to touch between two neighbor spots. The latter time value can be in turn considered as a threshold time in order to avoid edge-to-edge interference. We can then empirically suggest that the step time is set at approximately $1 / 2$ of this time length to attain accurate results. The approach is confirmed by the simulations (see inset in Figure 3): the diffusion profiles are well separated after $3 \mathrm{~s}$.

In order to test the validity of the optimized waveform for the real samples, a series of measurements were performed above the spot made by $\mathrm{IrO}_{2} 100 \%$. After this, and thanks to knowledge of each spot position (determined by linear scans before the actual screening experiment), all of the other spots were individually addressed at the same distance. Figure 4

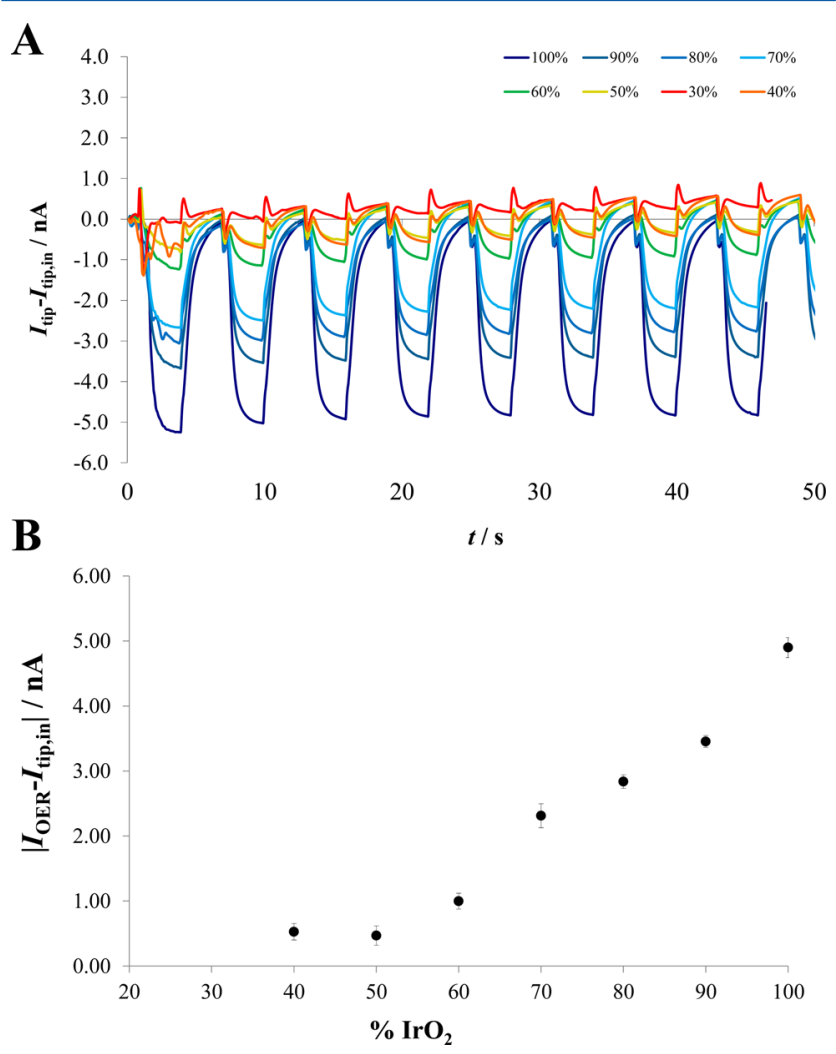

Figure 4. SG/TC double-pulse technique applied to several spots in the $\mathrm{IrSn} 1$ array in $0.5 \mathrm{M} \mathrm{H}_{2} \mathrm{SO}_{4}$. In this case, $E_{\text {rest }}=0.9 \mathrm{~V}$ and $E_{\mathrm{OER}}=$ $1.4 \mathrm{~V}$ and tip-to-spot distance $d=20 \mu \mathrm{m}$. (A) Background-subtracted tip current plotted in the ordinate and time in the abscissa. Color coding indicates the percentage of $\mathrm{Ir}$ in each spot. (B) Maximum background subtracted current versus $\% \mathrm{IrO}_{2}$ content.

shows a series of tip current profiles thus obtained. After the application of the potential step, the current rises steeply, while it quickly decays to almost the background level after the square pulse ends.

The maximum current achieved at the end of the $E_{\mathrm{OER}}$ pulse, which is proportional to the local oxygen concentration generated over the spot, was considered for evaluating the activity of the material. The average value obtained from eight consecutive applied pulses allowed also to verify the reproducibility of the signal. However, we noticed that during repetitive application of several series of pulses there was a progressive drift of the tip current at both currents resulting from biasing to at $E_{\mathrm{OER}}$ and $E_{\text {rest. }}$. This was likely due to a 
progressive poisoning of the $\mathrm{Au}$ surface, an effect often observed in SECM experiments. Notwithstanding this, it was always possible to screen series of spots without cleaning the tip and the drift is compensated by correcting the tip current for its value read at steady state before the substrate potential is pulsed.

3.2. Screening of the SnIrOx Array. In order to carry out the double-pulse methodology, we addressed each spot individually. To do this, the first step consists of the identification of the coordinates of each spot in the array by carrying out linear scans or a $x y$ image at constant distance. Here, we used the SG/TC mode, collecting $\mathrm{O}_{2}$ at the tip while the array was biased at $1.5 \mathrm{~V}$ vs RHE. At this potential, all spots are fairly active and it is easy to identify their location.

Figure 4 represents a collection of tip current transients recorded over several spots on the SnIr1 array. In this case, the substrate potential was pulsed between $E_{\text {rest }}=0.9 \mathrm{~V}$ and $E_{\mathrm{OER}}=$ $1.4 \mathrm{~V}$ vs RHE, and the current values displayed are relative to the initial current value recorded before the application of the pulse, $I_{\text {tip,in. }}$. As shown in Figure 4A, the tip current follows the double pulse profile (activity/rest) applied to the substrate. Spots containing less than $30 \% \quad \mathrm{IrO}_{2}$ showed negligible production of oxygen.

We analyzed the peak current under oxygen evolution $\left(I_{\text {tip,OER }}\right)$, subtracted for the initial background current $\left(I_{\text {tip,in }}\right.$, i.e., after the stabilization time) for each spot composition. The results are summarized in Figure $4 \mathrm{~B}$ and clearly identify an increase in $\% \mathrm{IrO}_{2}$ with an increase in sample activity toward OER. This trend is approximately linear and is consistent with previously reported SECM SG/TC of the same reacting system using a metal-shielded SECM tip. ${ }^{27}$ In contrast to that work, the approach reported here affords the simplicity of using a conventional SECM tip. Although it might seem inconvenient to address every spot individually, we found that this activity resulted in a similar time and effort expenditure than that typically invested in SG/TC imaging methods.

The rest potential has an impact on the shape of the current transients. Unlike the current read under $E_{\mathrm{OER}}$, which reached a steady-state value for most compositions, for the lowest active spots a charging current contribution from the potential switch is visible as spikes that appear just after the application of the potential step. This effect was dependent on the $\Delta E$. To prove this, we also carried out measurements at $E_{\text {rest }}$ i.e., $1.2 \mathrm{~V}$ (RHE), and these results are summarized in Figure 5.

The two sets of measurements $\left(E_{\text {rest }}=0.9\right.$ or $\left.1.2 \mathrm{~V}\right)$ are in agreement for what concerns the activity of the spots. Minor discrepancies might derive from slight differences in the tip positioning before the analysis of each single spot. When $\Delta E=$ $E_{\mathrm{OER}}-E_{\text {rest }}$ is made smaller, it results in a lower incidence of spikes due to a lower capacitive change. The absence of spikes allows us to better discuss the shape of the transients. In particular, at $E_{\mathrm{OER}}$ the $I_{\text {tip }}$ almost reaches the steady state, whereas at $E_{\text {rest }}$ the tip current transient is slower. The main difference in the two situations is due to the different diffusion profile shape transients. At $E_{\mathrm{OER}}$, the substrate produces oxygen that is rapidly reduced at the tip. In other words, the system is now in a substrate generation-tip collection (SG-TC) SECM configuration: oxygen diffuses from the spot to the tip forming a quasi-hemispheric profile and a steady state is rapidly reached. On the other side, i.e., at $E_{\text {rest }}$ the tip reduces oxygen coming from the solution and the diffusion front moves from the space between the metallic part of the tip and the electrocatalytic spot to the volume between the tip glass and the substrate, thus

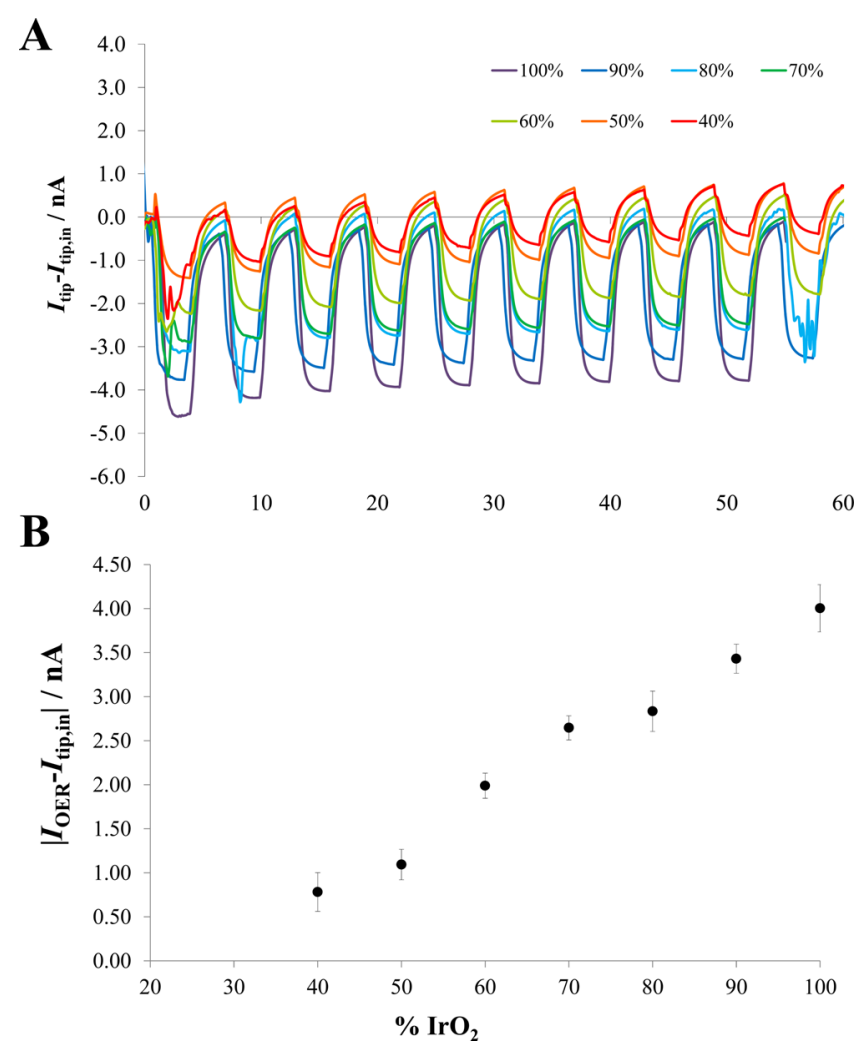

Figure 5. SG/TC double-pulse technique applied to several spots in the $\mathrm{IrSn} 1$ array in $0.5 \mathrm{M} \mathrm{H}_{2} \mathrm{SO}_{4}$. In this case, $E_{\text {rest }}=1.2 \mathrm{~V}$ and $E_{\mathrm{OER}}=$ $1.4 \mathrm{~V}$ and tip-to-spot distance $d=20 \mu \mathrm{m}$. (A) Background subtracted tip current plotted in the ordinate and time in the abscissa. Color coding indicates the percentage of $\mathrm{Ir}$ in each spot. (B) Maximum background subtracted current versus $\% \mathrm{IrO}_{2}$ content.

assuming a planar, semi-infinite profile. In this last case, reaching a steady state requires more time. Finally, it is noteworthy that all measurements here were carried out in the presence of atmospheric oxygen dissolved in the solution. Because the present technique allows a very accurate measurement of the activated and background current, it is possible to confidently subtract the background contributions. The double pulse technique presented in here removes some of the more experimentally cumbersome aspects of SG/TC mechanistic investigations such as substrate tilt leveling, specialized tips for avoiding interferences, slow imaging rates, uncertainties in spot localization and the removal of atmospheric contaminations such as oxygen.

As for the time needed for screening an array of spots in comparison to a "conventional" imaging experiment, we estimate that, for a $3 \times 11$ spot array, imaging can require about $3-5 \mathrm{~h}$, which include $1-2 \mathrm{~h}$ for tilting correction, while the proposed method reduces preparation time to about $1 \mathrm{~h}$, after which the analysis of each spot requires no more than 5 min. In our case, the total experimentation time was $4 \mathrm{~h}$. Therefore, the time frame is slightly shorter, and although in the "conventional" method one could cut-back on experimental time by introducing improvements such as motorized tilt correction, this instrumentation is not yet widely available in commercial SECM instruments.

Thus, the new proposed method should be useful for studying the mechanisms of electrocatalytic reactions on arrays with sample sizes of hundreds of micrometers, as it is currently allowed by microdispenser techniques. 


\section{CONCLUSIONS}

In this paper, the screening of electrocatalysts by a substrate potential double-pulse method of SECM was introduced, discussed, and applied on an array made of $\mathrm{Sn}-\mathrm{Ir}$ oxides spots for the oxygen evolution reaction. Previous uses of pulsed substrate potential profiles have been proposed in the literature, ${ }^{19,22}$ but the present work introduces this approach for the screening of arrays by means of individually addressing each catalyst spot. The method is designed to operate in the substrate generation/tip collection mode of SECM, of which a main obstacle is the partial overlap of diffusion profiles from neighboring spots when the sample consists of a geometric array of active compositions. This overlap causes the tip current to misrepresent the activity of the spot underneath. By selectively alternating between a potential where the samples are active and where they rest using a square potential waveform, the diffusive overlap can be suppressed by choosing a pulse time well below the characteristic diffusive time, $\tau=d^{2}$ / $2 D$.

Here, we demonstrated by both digital simulations and experiments onto a "standard" array, that the method proved effective in obtaining a correct ranking of catalyst activity. The method proved also to be rapid since it does not require neither the precise adjustment of the substrate tilting nor the saturation of the electrolyte with an inert gas.

We believe that the method here described will not only be useful for the SG/TC mode of SECM but can also be extended to multipurpose catalysts where cleaning or preconditioning steps are necessary for studying reaction mechanisms. In addition, the study of photoelectrodes could be improved by this approach, for example, in the screening of semiconductors $^{31}$ or of photoelectrocatalysts. ${ }^{12}$ The possibility of using a nontilted substrate is a great advantage in the case of substrates that are intrinsically not flat or bent because of a nonoptimal cell housing. In addition, the possibility of using an ordinary SECM tip makes the method particularly robust and even more rapid, since the preparation of $\mathrm{Au}$ coated tips can require up to 3 days.

\section{AUTHOR INFORMATION}

\section{Corresponding Author}

*Tel: +390250314224. E-mail: alessandro.minguzzi@unimi.it.

\section{Notes}

The authors declare no competing financial interest.

\section{ACKNOWLEDGMENTS}

This material is based upon work supported by MIUR (Futuro in Ricerca 2013, project RBFR13XLJ9) and by the National Science Foundation (Grant No. CHE-1405248).

\section{REFERENCES}

(1) Fernandez, J. L.; Bard, A. J. Scanning electrochemical microscopy. 47. imaging electrocatalytic activity for oxygen reduction in an acidic medium by the tip generation-substrate collection mode. Anal. Chem. 2003, 75, 2967-2974.

(2) Fernandez, J. L.; Bard, A. J. Scanning electrochemical microscopy 50. Kinetic study of electrode reactions by the tip generationsubstrate collection mode. Anal. Chem. 2004, 76, 2281-2289.

(3) Jung, C.; Sanchez-Sanchez, C. M.; Lin, C.-L.; Rodriguez-Lopez, J.; Bard, A. J. Electrocatalytic activity of Pd-Co bimetallic mixtures for formic acid oxidation studied by scanning electrochemical microscopy. Anal. Chem. 2009, 2009, 7003-7008.
(4) Wain, A. J. Imaging size effects on the electrocatalytic activity of gold nanoparticles using scanning electrochemical microscopy. Electrochim. Acta 2013, 92, 383-391.

(5) Weng, Y.-C.; Hsieh, C.-T. Scanning electrochemical microscopy characterization of bimetallic $\mathrm{Pt}-\mathrm{M}(\mathrm{M}=\mathrm{Pd}, \mathrm{Ru}, \mathrm{Ir})$ catalysts for hydrogen oxidation. Electrochem. Acta 2010, 56, 1932-1940.

(6) Zeradjanin, A. R.; Menzel, N.; Schuhmann, W.; Strasser, P. On the faradaic selectivity and the role of surface inhomogeneity during the chlorine evolution reaction on ternary $\mathrm{Ti}-\mathrm{Ru}-\mathrm{Ir}$ mixed metal oxide electrocatalysts. Phys. Chem. Chem. Phys. 2014, 16, 1374113747.

(7) Rodriguez-Lopez, J.; Alpuche-Aviles, M. A.; Bard, A. J. Selective insulation with poly(tetrafluoroethylene) of substrate electrodes for electrochemical background reduction in scanning electrochemical microscopy. Anal. Chem. 2008, 80, 1813-1818.

(8) Sanchez-Sanchez, C. M.; Bard, A. J. Hydrogen peroxide production in the oxygen reduction reaction at different electrocatalysts as quantified by scanning electrochemical microscopy. Anal. Chem. 2009, 81, 8094-8100.

(9) Mirkin, M. V.; Nogala, W.; Velmurugan, J.; Wang, Y. Scanning electrochemical microscopy in the 21st century. Update 1: five years later. Phys. Chem. Chem. Phys. 2011, 13, 21196-21212.

(10) Bard, A. J. Inner-sphere heterogeneous electrode reactions. electrocatalysis and photocatalysis: the challenge. J. Am. chem. Soc. 2010, 132, 7559-7567.

(11) Lin, C.-L.; Rodriguez-Lopez, J.; Bard, A. J. Micropipette delivery-substrate collection mode of scanning electrochemical microscopy for the imaging of electrochemical reactions and the screening of methanol oxidation electrocatalysts. Anal. Chem. 2009, 81, 8868-8877.

(12) Rodriguez-Lopez, J.; Zoski, C. G.; Bard, A. J.: SECM applications to electrocatalysis and photocatalysis and surface interrogation. In Scanning Electrochemical Microscopy, 2nd ed.; Bard, A. J., Mirkin, M. V., Eds.; Marcel Dekker: New York, 2012; pp 525568.

(13) Johnson, L.; Walsh, D. A. Tip generation-substrate collectiontip collection mode scanning electrochemical microscopy of oxygen reduction electrocatalysts. J. Electroanal. Chem. 2012, 682, 45-52.

(14) Eckhardt, K.; Chen, X.; Turcu, F.; Schuhmann, W. Redox competitiom mode of scanning electrochemical microscopy (RCSECM) for visualization of local catalytic activiy. Phys. Chem. Chem. Phys. 2006, 8, 5359-5365.

(15) Guadagnini, L.; Maljusch, A.; Chen, X.; Neugeauer, S.; Tonelli, C.; Schuhmann, W. Visualization of electrocatalytic activity of microstructured metal hexacyanoferrates by means of redox competetion mode of scanning electrochemical microscopy (RCSECM). Electrochim. Acta 2009, 54, 3753-3758.

(16) Sanchez-Sanchez, C. M.; Rodriguez-Lopez, J.; Bard, A. J. Scanning Electrochemical Microscopy. 60. Quantitative calibration of the SECM substrate generation/tip collection mode and its use for the study of the oxygen reduction mechanism. Anal. Chem. 2008, 80, 3254-3260.

(17) Martin, R. D.; Unwin, P. R. Scanning electrochemical microscopy. Kinetics of chemical reactions following electron-transfer measured with the substrate-generation-tip-collection mode. J. Chem. Soc., Faraday Trans. 1998, 94, 753-759.

(18) O'Mullane, A. P.; Neufeld, A. K.; Bond, A. M. Monitoring cuprous iion transport by scanning electrochemical microscopy during the course of copper electrodeposition. J. Electrochem. Soc. 2008, 155, D538-D541.

(19) Shen, Y.; Trauble, M.; Wittstock, G. Detection of hydrogen peroxide produced during electrochemical oxygen reduction using scanning electrochemical microscopy. Anal. Chem. 2008, 80, 750-759.

(20) Mezour, M. A.; Renaud, C.; Hussien, E. M.; Morin, M.; Mauzeroll, J. Detection of hydrogen peroxide produced during the oxygen reduction reaction at self-assembled thiol-porphyrin monolayers on gold using SECM and nanoelectrodes. Langmuir 2010, 26, 13000-13006. 
(21) Rodriguez-Lopez, J.; Ritzert, N. L.; Mann, J. A.; Tan, C.; Dichtel, W. R.; Abruna, H. D. Surface diffusion of electrochemically active tripodal motifs on graphene, a scanning electrochemical microscopy approach. J. Am. Chem. Soc. 2012, 134, 6224-6236.

(22) Eckhardt, K.; Schuhmann, W. Localized visualization of O2 consumption and $\mathrm{H} 2 \mathrm{O} 2$ formation by means of SECM for the characterization of fuel cell catalyst activity. Electrochem. Acta 2007, 53, $1164-1169$.

(23) Nogala, W.; Burchardt, M.; Opallo, M.; Rogalski, J.; Wittstock, G. Scanning electrochemical microscopy study of laccase within a solgel processed silicate film. Bioelectrochemistry 2008, 72, 174-182.

(24) Li, F.; Su, B.; Salazar, F. C.; Nia, R. P.; Girault, H. H. Detection of hydrogen perxode produced at a liquid/liquid interface using scanning electrochemical microscopy. Electrochem. Commun. 2009, 11, 473-476.

(25) Maljusch, A.; Edgar, V.; Rincon, R. A.; Bandarenka, A. S.; Schuhmann, W. Revealing onset potentials using electrochemical microscopy to assess the catalytic activity of gas-evolving electrodes. Electrochem. Commun. 2014, 38, 142-145.

(26) Zeradjanin, A. R.; Schilling, T.; Seisel, S.; Bron, M.; Schuhmann, $\mathrm{W}$. Visualization of chlorine evolution at dimensionally stable anodes by means of scanning electrochemical microscopy. Anal. Chem. 2011, $83,7645-7650$.

(27) Minguzzi, A.; Alpuche-Aviles, M. A.; Rodriguez-Lopez, J.; Rondinini, S.; Bard, A. J. Screening of oxygen evolution electrocatalysts by scanning electrochemical microscopy using a shielded tip approach. Anal. Chem. 2008, 80, 4055-4064.

(28) Snook, G. A.; Duffy, N. W.; Pandolfo, A. G. Detection of oxygen evolution from nickel hydroxide electrodes using scanning electrochemical microscopy. J. Electrochem. Soc. 2008, 155, A262-A267.

(29) Skylar, O.; Traeuble, M.; Zhao, C.; Wittstock, G. Modeling steady-state experiments with a scanning electrochemical microscope involving several independent diffusing species using the boundary element method. J. Phys. Chem. B 2006, 110, 15869-15877.

(30) Mirkin, M. V. In Scanning Electrochemical Microscopy; Bard, A. J., Mirkin, M. V., Eds.; Marcel Dekker Inc.: New York, 2001; pp 145199.

(31) Minguzzi, A.; Sánchez-Sánchez, C. M.; Gallo, A.; Montiel, V.; Rondinini, S. Evidence of facilitated electron transfer on hydrogenated self doped $\mathrm{TiO}_{2}$ nanocrystals. ChemElectroChem 2014, 1, 1415-1421. 